\title{
Peritoneal ultrafiltration in end-stage congestive heart failure
}

\author{
Ewa Wojtaszek ${ }^{1}$, Jolanta Małyszko², Joanna Matuszkiewicz-Rowińska ${ }^{1}$ \\ ${ }^{1}$ Department and Clinic of Nephrology, Dialysis and Internal Diseases, Medical University of Warsaw, Poland \\ ${ }^{2} 2^{\text {nd }}$ Department of Nephrology, Medical University of Bialystok, Poland
}

\begin{abstract}
Congestive heart failure (CHF) refractory to pharmacological therapy is a growing medical problem. Renal sodium and water retention remains a key event in the pathogenesis of the disease progression and episodes of severe cardiac decompensation, being also the leading cause of high hospitalization rates and an important risk factor for worsening kidney function and chronic kidney disease (CKD). The two conditions: CHF and CKD form a vicious circle, with a tremendous escalation of complications and mortality. In this clinical situation, peritoneal ultrafiltration (PUF) may be a reasonable choice for long-term treatment of selected patients with end-stage CHF, especially for those with contradictions to heart transplantation. Several observational studies have demonstrated its efficacy and safety in this population. Fluid and sodium removal via peritoneal cavity resulted in significant plasma volume reduction, normalization of serum sodium and restoration of diuretic responsiveness, as well as an improvement in New York Heart Association functional class, reduction of hospitalization and readmission rates. The complications are typical for peritoneal dialysis (catheter exit site infections, peritonitis and fluid leaks) but they are much more rare with 1 instead of 4 exchanges per day, and it seems that at least in case of PUF with 1-2 peritoneal exchanges a day, the benefits of the therapy outweigh the risks. However, as the studied groups were small, larger multicenter randomized trials are necessary to develop precise recommendations regarding clinical aspects of PUF in severe CHF and indications for its use. (Cardiol J 2014; 21, 2: 115-120)
\end{abstract}

Key words: congestive heart failure, chronic kidney disease, ultrafiltration, peritoneal dialysis

\section{Introduction}

It is estimated that $1-2.5 \%$ of adult population suffers from congestive heart failure (CHF), while in patients over 70 years of age this proportion exceeds $10 \%$ [1]. Although the last decades brought a considerable improvement in the therapy of patients with CHF, leading to a reduction in their mortality and morbidity, the long-term prognosis remains poor [2].

Advanced CHF is often accompanied by varying degrees of chronic kidney disease (CKD).
Coexistence of both pathologies is referred to as cardiorenal syndrome type 2 [3]. Reduced cardiac output leads to a reduction in the glomerular filtration rate (GFR), a strong stimulus activating neurohormonal adaptation axes (renin-angiotensin-aldosterone, noradrenergic system, vasopressin secretion) with renal sodium and water retention, simultaneously persisting renal hypoperfusion with an increase in renal vascular resistance stimulating the development of progressive ischemic nephropathy. On the other hand, consequences of CKD that appear as the disease advances, such

Address for correspondence: Prof. Jolanta Małyszko, $2^{\text {nd }}$ Department of Nephrology, Medical University of Bialystok, ul. Żurawia 14, 15-540 Białystok, Poland, e-mail: jolmal@poczta.onet.pl

Received: 17.02.2013

Accepted: 25.06.2013 
as: anemia, inflammation and cardiovascular calcifications, contribute considerably to progression of CHF. A vicious cycle is induced, in which each condition (CHF and CKD) exacerbates and stimulates the progression of the other. Heart failure usually advances more rapidly, and constitutes the leading cause of death before the development of uremia requiring renal replacement therapy $[2,3]$.

About $5 \%$ of patients with CHF progress to its end-stage stadium. Sodium and water retention, fundamental to this progression, remains also one of the main causes $(>90 \%)$ of hospitalizations $[2,4]$. End-stage CHF is characterized by a significant resistance to pharmacological treatment, especially to diuretics. This is caused, among other things, by an impairment of drug delivery to renal tubules due to both hypoperfusion and edema of kidneys, which disrupts the hemodynamic balance in the renal glomerulus with a significant reduction in GFR [5, 6]. In order to overcome this resistance, a transient use of intravenous loop diuretics is necessary. Unfortunately, it is not always fully effective and often leads to worsening the kidney functioning. In about $40 \%$ of patients, at the time of discharge from hospital fluid overload of varying degrees persists and almost $30 \%$ of them are readmitted within a month $[4,7]$. Mortality in this group is dramatically high; it is estimated to reach $40-50 \%$ within 6 months $[2,7,8]$. Unfortunately, due to advanced age and comorbidities, most of these patients have contraindications to non-pharmacological treatment, such as heart transplantation, resynchronization therapy or other surgical methods. Therefore, it is of great importance to establish a treatment strategy that would ensure the most effective volume reduction without the adverse influence on renal function and patients hemodynamics, improving patient's quality of life by reducing symptoms and frequency of hospitalizations, and - maybe - giving a chance for better prognosis.

\section{Ultrafiltration}

In some patients with considerable fluid overload and significant loop diuretic resistance, ultrafiltration (UF) may be the only effective method of removing the excess fluid. Such a purely mechanical method of reducing intravascular volume leads to lowering the systemic venous pressure as well as the pressure in pulmonary circulation accompanied by improvement in hemodynamic parameters and decreased adverse influence of neurohormonal activation on cardiac and renal function. It has been demonstrated, that in patients with $\mathrm{CHF}$ receiving
UF, plasma renin, norepinephrine and aldosterone concentrations are lower as long as 90 days after treatment compared with those receiving diuretics [9]. Simultaneously, UF improves hemodynamic conditions within the renal glomerulus by reducing parenchymal edema, accompanied by GFR elevation, increased diuresis and restoration of sensitivity to diuretics [9-11]. In a study by Guazzi et al. [12] of 22 patients with CHF, the use of UF was associated with a significant diuresis increase ( $852 \mathrm{vs.} 2132 \mathrm{~mL} / \mathrm{d}$ ) as well as urinary sodium excretion (39 vs. $137 \mathrm{mmol} / \mathrm{d}$ ) $24 \mathrm{~h}$ after the therapy.

In comparison to diuretic therapy, UF is more effective in net sodium removal, as the ultrafiltrate is isonatremic and iso-osmolar compared with plasma as opposed to the hypotonic urine produced in response to diuretics [13]. Therefore, UF removes more sodium and less potassium, allowing to avoid electrolyte disturbances often observed during treatment with diuretics. Finally, contrary to loop diuretics, which increase sodium influx into the distal tubule through inhibition of $\mathrm{Na}-\mathrm{K}-2 \mathrm{Cl}$ channels, UF — sparing exposure of the renal tubules to sodium - does not trigger to such extent the maladaptive tubule-glomerular feedback with paracrine effects on local vasculature $[6,14]$.

There are two types of UF: extracorporeal and peritoneal.

\section{Extracorporeal ultrafiltration}

Extracorporeal UF performed with an artificial kidney utilizes the mechanism of water removal from plasma through a semipermeable membrane due to the transmembrane pressure gradient. For many years, the so-called isolated extracorporeal UF has been used in dialysis centers for various extrarenal reasons, particularly in patients with massive generalized edema, usually occurring in the course of nephrotic syndrome. In acute CHF decompensation it may be a life-saving treatment method; it can also contribute to shortening the hospitalization time [10, 15-17]. However, extracorporeal UF is associated with the need to gain vascular access, administration of systemic anticoagulation, use of special devices (artificial kidney, filters, bloodlines), and presence of qualified medical personnel. Moreover, it may be conducted exclusively in a hospital environment or even in intensive care units in case of hemodynamically unstable patients. Excessively rapid fluid removal from the intravascular space may induce episodes of severe hypotension with organ hypoperfusion, including central nervous system, heart or kidneys. 
While this method may be useful in patients with severe cardiovascular decompensation, its long-term use may be problematic. That would mean either the necessity of permanent hospitalization or performing the procedure 3-4 times a week, as in case of chronic dialysis for end-stage renal disease. It poses a risk of significant fluctuations in volume status with augmentation of heart failure symptoms between treatments and hypotension during the procedure, hindering adequate water removal. These phenomena are often observed in chronically hemodialyzed patients and are thought to contribute considerably to progression of heart disease. Peritoneal ultrafiltration (PUF) with or without peritoneal dialysis (PD) provides a chance to avoid such problems.

\section{Peritoneal ultrafiltration}

Chronic PD is a renal replacement therapy conducted by the patient (or a family member) under home conditions. Due to a specific structure of densely vascularized peritoneum, it takes advantage of the fact that, after filling peritoneal cavity with an osmotically active fluid (containing glucose, amino acids or icodextrin) molecules with small- or medium-molecular mass (through diffusion) and water (through convection) pass from patient's blood into the peritoneal cavity.

Effectiveness of both processes depends on patient-specific properties of peritoneal membrane on one hand, but on the other, it may be widely modified through various methods of dialysis fluid application (manual or automatic), its volume, frequency of exchanges as well as the type and concentration of an osmotically active substance. Fluids for PD do not contain potassium, which minimizes the risk of hyperkalemia and allows optimization of therapy with angiotensin converting enzyme inhibitors or an aldosterone antagonists. Continuous slow character of PUF does not cause significant blood volume fluctuations and facilitates hemodynamic stability, while constant evacuation of fluid from the peritoneal cavity maintains intra-abdominal pressure at a relatively low level, allowing to sustain the response to diuretics and preserve the renal function. Additionally, a potential benefit may involve transperitoneal removal of inflammatory molecules and other cardio-depressants, improving myocardial function, as well as prevention of ascites, minimizing discomfort, and lowering the risk of spontaneous bacterial peritonitis $[11,18]$. Nevertheless, these issues need further investigation.
Dialysis fluids containing icodextrin may be of particular interest with regard to treatment of patients with heart failure. It is a high-molecular mass (about $17 \mathrm{kDa}$ ) glucose polymer, iso-osmolar to blood plasma, which induces UF through colloidal osmosis. Due to minimal diffusion into the systemic circulation it leads to constant high UF regardless of peritoneal membrane transportation properties. So far it has been the only substance that can provide UF when it stays in the peritoneal cavity for longer than $8 \mathrm{~h}$. Experimental treatments in patients with end-stage renal disease indicate that icodextrin is more effective than glucose with regard to systemic extravascular water reduction and this effect is proportional the to state of hydration [19]. It is also more effective in removing medium-size compounds (e.g. leptin) and finally, as opposed to glucose, does not cause metabolic disorders (hyperinsulinemia, dyslipidemia) [19].

Peritoneal dialysis was first successfully used in treatment of heart failure over 60 years ago [20]. Since then, several case reports and case series have been published, demonstrating the beneficial effects of $\mathrm{PD} / \mathrm{PUF}$ in patients with severe treatment-resistant $\mathrm{CHF}$, regardless of the degree of renal impairment. Unfortunately, they are uncontrolled and the groups studied are small. Patients with coexisting renal failure may be treated with intermittent PD [21], continuous ambulatory PD [22-26] or automated PD [27-30], while in those without significant impairment of renal function - a single nightly exchange (PUF) with osmotic agent (optimally icodextrin) is sufficient [31-35]. In the vast majority of cases PD/PUF was performed in patients with advanced CHF and symptoms of cardiorenal syndrome as an addition to standard therapy. It usually concerned elderly patients with numerous comorbidities and various degrees of renal impairment, disqualified from heart transplantation and refractory to pharmacological treatment. In some centers, extracorporeal UF was initially performed for quick reduction of fluid overload [28, 29, 32-35], while in the others therapy was commenced with PUF or PD. PUF/PD dose was matched to individual patient's needs in order to obtain optimal UF on one hand, while achieving adequate balancing of metabolic disturbances associated with renal impairment on the other.

The most important clinical studies on PD or an isolated PUF are presented in Table 1 . The benefits obtained were similar regardless of the applied method (PUF or PD), although different studies analyzed various parameters. Commencement of PUF/PD was associated with improved response 


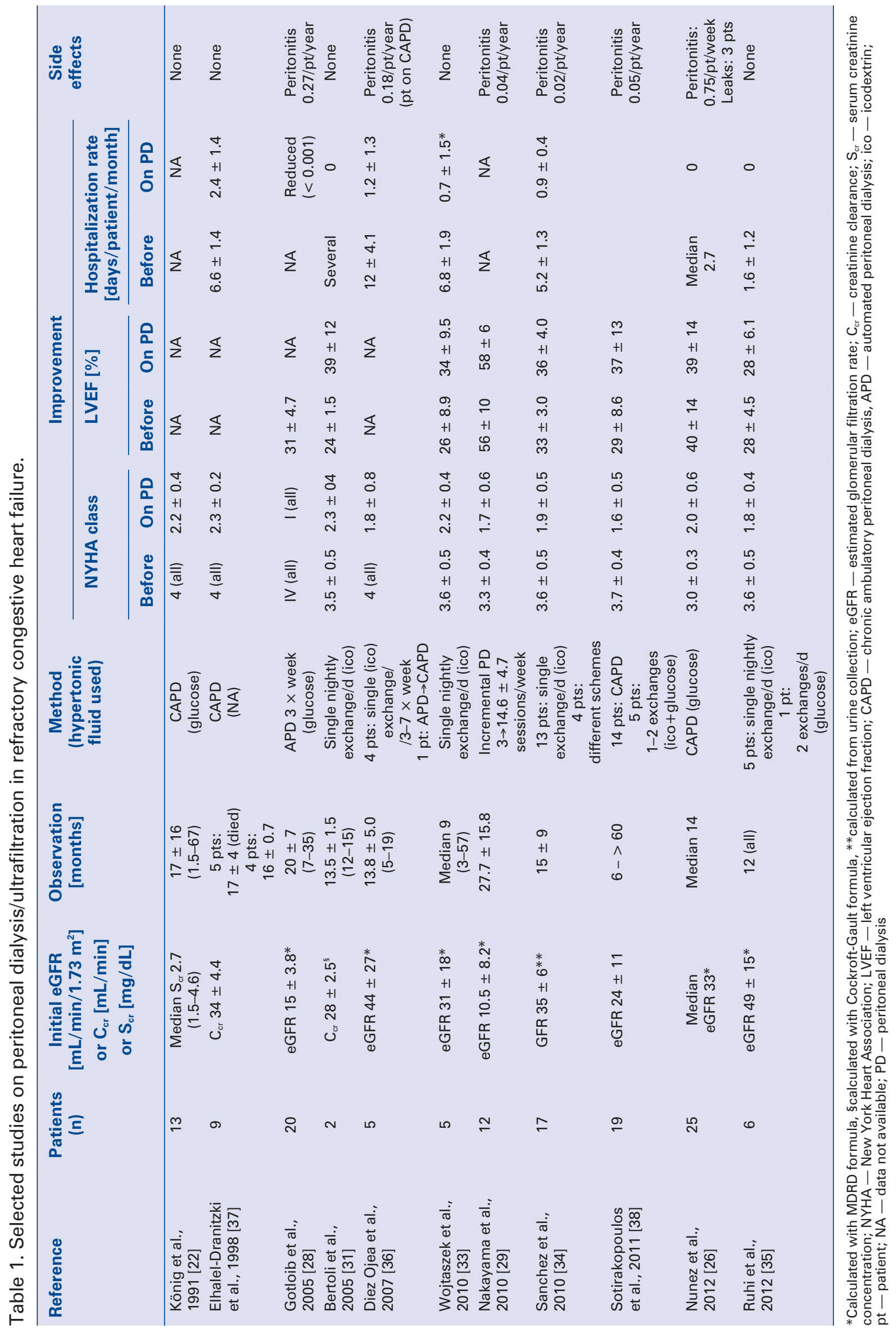


to diuretics, allowing for a significant (by as much as $50 \%$ ) dose reduction and increased diuresis with stable renal function during the observation period [26, 29, 32-35]. The effect of continuous, slow removal of water and sodium excess via peritoneal cavity may be of significance here, as it facilitates gradual reduction of extracellular volume and, subsequently, maintenance of normovolemia and neurohormonal stabilization. That would be indicated by, as observed by some authors, a decrease in plasma renin activity, as well as in serum concentrations of aldosterone and natriuretic peptides: atrial and B-type [22, 29]. Reduction or disappearance of clinical symptoms, accompanied by functional improvement in New York Heart Association (NYHA) class [22, 23, 25, 28, 30, 31, 33,34 , with better exercise capacity [26] was observed in a great majority of patients. In some reports, this spectacular clinical improvement was accompanied by an increase in heart ejection fraction $[25,28,31,34]$, or even - by a reduction in pulmonary artery pressure [24, 26, 34], which could be of great significance, giving some patients a chance to re-qualify for heart transplantation [22, 23]. In such patients PUF could constitute a "bridge therapy" while awaiting heart transplantation, simultaneously reducing the risk of organ damage that could disqualify them from such a procedure.

The spectacular, 6-12-fold reduction in the frequency and duration of hospitalization, remains a fundamental advantage of PUF/PD [23, 24, 26-28, $30-34]$. This effect is particularly significant in patients who use it as palliative therapy with regard to their quality of life as well as healthcare costs $[26,34]$. Pharmacoeconomic analysis conducted by authors from Spain revealed a nearly 4 -fold reduction in yearly costs with application of PUF in comparison to standard therapy [34].

PUF is a relatively safe therapy. The potential side effects are the same as for standard PD, including catheter related infections and mechanical problems (fluid leaks), but they are much more rare with 1 or 2 exchanges per day [23, 28, 29, 32-34]. The use of 1 instead of 4 peritoneal exchanges reduced significantly the risk of peritonitis and exit-site infections. In the case series of 17 patients treated by PUF reported by Sanchez et al. [34], peritonitis was the only technique complication, being 28 times rarer than in Spanish PD Unit Registry (0.02 vs. 0.56 episodes/patient/year at risk; $\mathrm{p}=0.008)$. Mechanical problems (leaks, hernias) are mostly related to the increase of intra-abdominal pressure during the exchange. Pericatheter leaks usually occur after an acute start of PD, and they can be
Table 2. Contradictions to peritoneal ultrafiltration.

- History of abdominal surgeries with extensive
adhesions or ostomies
- Patient's/caregiver's incapability of performing
the procedure, poor hygiene
- Uncorrectable mechanical defects that increase
the risk of complications (e.g. surgically
irreparable hernia)
- Abdominal abscess
- Severe inflammatory bowel disease, active
ischemic bowel disease, recurrent diverticulitis
- Morbid obesity
- Advanced chronic obstructive pulmonary
disease
- Extensive abdominal wall infection
- Recent history (4-6 months) of abdominal
vascular surgery with prostheses

prevented by delaying PUF for 10-14 days after catheter insertion. Before starting the patient on PUF the contradictions to PD must be ruled out (Table 2), and a careful search for a presence of hernias has to be performed, which, if found, should be repaired. In many cases it can be done simultaneously with catheter insertion under local anesthesia.

It is difficult to say whether broadening the standard therapy to include PUF would significantly change the natural course of the disease and improve patients' survival. In a recent analysis of Polish Registry of Patients Referred for Heart Transplantation (POLKARD HF) only symptoms compatible with NYHA class IV, persisting despite an adequate treatment were related to a bad prognosis [8]. However, available data acquired from non-randomized trials on small numbers of patients and with short observation times do not allow drawing any conclusions. It is only possible to suggest that, since a 6 -month mortality among patients resistant to treatment exceeds $50 \%$, any method that could potentially reverse this dramatically poor prognosis is highly desirable [2, 7]. In this context, yearly mortality of $10-15 \%$ reported by some authors in a group of patients treated with PUF $[28,30]$ should be considered a satisfactory improvement in survival. Undoubtedly, this effect should be assessed in controlled, randomized clinical trials.

\section{Conclusions}

All the data gathered to date from observational studies on the role of PUF as palliative treatment adjuvant to standard pharmacotherapy 
in patients with severe CHF refractory to optimal treatment are encouraging and indicate their efficacy as well as safety. Given that the complications such as peritonitis or leaks are relatively rare, it seems that the benefits of the therapy far outweigh its risks. The greatest benefit is, according to the previous studies, improved quality of live as measured by spectacular reduction in the frequency of hospitalizations. Only the results of multicenter, randomized trials may answer the question whether PUF could extend patient's lifespan. It is also important to determine which patients could potentially benefit from this type of therapy the most and what could be the value of this method as a "bridge to transplantation" therapy.

\section{Conflict of interest: none declared}

\section{References}

1. Rosamond W, Flegal K, Furie K et al.; for American Heart Association Statistics Committee and Stroke Statistics Subcommittee. Heart disease and stroke statistics 2008 update: A report from the American Heart Association Statistics Subcommittee. Circulation, 2008; 117: e25-e146.

2. Laribi S, Aouba A, Nikolaoul M et al. Trends in death attributed to heart failure over the past two decades in Europe. Eur J Heart Fail, 2012; 14: 234-239.

3. Ronco C, Harpio M, House AA, Anavekar N, Bellomo R. Cardiorenal syndrome. J Am Coll Cardiol, 2008; 52: 1527-1539.

4. Gheorghiade M, Follath F, Ponikowski P et al. Assessing and grading congestion in acute heart failure: a scientific statement from the Acute Heart Failure Committee of the Heart Failure Association of the European Society of Cardiology and endorsed by the European Society of Intensive Care Medicine. Eur J Heart Fail, 2010; 12: 423-433.

5. Chiong JR, Cheung RJ. Loop diuretic therapy in heart failure: The need for solid evidence on a fluid issue. Clin Cardiol, 2010; 33: 345-352.

6. Sarraf M, Masoumi A, Schirer RW. Cardiorenal syndromes in acute decompensated heart failure. Clin Am Soc Nephrol, 2009; 4: 2013-2026.

7. Jencks SF, Williams MV, Coleman EA. Rehospitalizations among patients in the Medicare fee-for-service program. N Engl J Med, 2009; 360: 1418-1428.

8. Korewicki J, Leszek P, Zielinski T et al. Severe chronic heart failure in patients considered for heart transplantation in Poland. Cardiol J, 2012; 19: 36-44.

9. Agostoni P, Marenzi G, Lauri G et al. Sustained improvement in functional capacity after removal of body fluid with isolated ultrafiltration in chronic cardiac insufficiency: Failure of furosemide to provide the same result. Am J Med, 1994; 96: 191-199.

10. Felker GM, Mentz RJ. Diuretics and ultrafiltration in acute decompensated heart failure. J Am Coll Cardiol, 2012; 59: 2145$-2153$.

11. Libetta C, Sepe V, Zucchi M et al. Intermittent haemodiafiltration in refractory congestive heart failure: BNP and balance of inflammatory cytokines. Nephrol Dial Transplant, 2007; 22: 2013-2019.

12. Guazzi MD, Agostoni P, Perego B et al. Apparent paradox of neurohumoral axis inhibition after body fluid volume depletion in patients with chronic congestive heart failure and water retention. Br Heart J, 1994; 72: 534-539.

13. Kamath SA. The role of ultrafiltration in patients with decompensated heart failure. Int J Nephrol, 2010; Epub 19 Oct.

14. Vallon V, Miracle C, Thomson S. Adenosine and kidney function: potential implications in patients with heart failure. Eur J Heart Fail, 2008; 10: 176-187.

15. Bart BA, Boyle A, Bank AJ et al. Ultrafiltration versus usual care for hospitalized patients with heart failure: The relief for acu- tely fluid-overloaded patients with decompensated congestive heart failure (RAPID-CHF) trial. J Am Coll Cardiol, 2005; 46: 2043-2046.

16. Costanzo MR, Guglin ME, Saltzberg MT et al.; for UNLOAD Trial Investigators. Ultrafiltration versus intravenous diuretics for patients hospitalized for acute decompensated heart failure. J Am Coll Cardiol, 2007; 49: 675-683.

17. Giglioli C, Landi D, Cecchi E et al. Effect of ultrafiltration vs. diuretics on clinical, biohumoral and haemodynamic variables in patients with decompensated heart failure: the ULTRADISCO study. Eur J Heart Fail, 2011; 13: 337-346.

18. Ross EA, Kazory A. Ultrafiltration therapy for cardiorenal syndrome: physiologic basis and contemporary options. Blood Purif, 2012; 34: 149-157.

19. Jörres A. Novel peritoneal dialysis solutions: What are the clinical implications? Blood Purif, 2012; 33: 153-159.

20. Schneierson SJ. Continuous peritoneal irrigation in the treatment of intractable edema of cardiac origin. Am J Med Sci, 1949; 218: 76-79.

21. Shapira J, Lang R, Jutrin I, Robson M, Ravid M. Peritoneal dialysis in refractory congestive heart failure. Part I: intermittent peritoneal dialysis. Perit Dial Bull, 1983; 3: 130-131.

22. Kőnig PS, Lhotta K, Kronenberg F, Joanidis M, Herold M. CAPD: A successful treatment in patients suffering from therapy-resistant congestive heart failure. Adv Perit Dial, 1991; 7: 97-101.

23. Ryckelynck JP, Lobbedez T, Valette B et al. Peritoneal utrafiltration and treatment-resistant heart failure. Nephrol Dial Transplant, 1998; 13 (suppl. 4): 56-59.

24. Kagan A, Rapoport J. The role of peritoneal dialysis in the treatment of refractory heart failure. Nephrol Dial Transplant, 2005; 20 (suppl. 7): 28-31.

25. Takane $\mathrm{H}$, Nakamoto $\mathrm{H}$, Arima $\mathrm{H}$ et al. Continuous ambulatory peritoneal dialysis for patients with severe congestive heart failure. Adv Perit Dial, 2006; 22: 141-146.

26. Núňez J, González M, Miňana G et al. Continuous ambulatory peritoneal dialysis as a therapeutic alternative in patients with advanced congestive heart failure. Eur J Heart Fail, 2012; 14: 540-548.

27. Ortiz AM, Acosta PA, Corbalan R, Jalil JE. Long-term automated peritoneal dialysis in patients with refractory congestive heart failure. Adv Perit Dial, 2003; 19: 77-80.

28. Gotloib L, Fudin R, Yakubovich M, Vienken J. Peritoneal dialysis in refractory end-stage congestive heart failure: a challenge facing a no-win situation. Nephrol Dial Transplant, 2005; 20: vii32-vii36.

29. Nakayama M, Nakano H, Nakayama M. Novel therapeutic option for refractory heart failure in elderly patients with chronic kidney disease by incremental peritoneal dialysis. J Cardiol, 2010; 55: 49-54.

30. Koch M, Haastert B, Kohnle $\mathrm{M}$ et al. Peritoneal dialysis relieves clinical symptoms ad is well tolerated in patients with refractory heart failure and chronic kidney disease. Eur J Heart Fail, 2012; 14: 530-539.

31. Bertoli SV, Ciurlino D, Maccario M et al. Home peritoneal utrafiltration in patients with severe congestive heart failure without end-stage renal disease. Adv Perit Dial, 2005; 21: 123-127.

32. Basile C, Chimienti D, Bruno A et al. Efficacy of peritoneal dialysis with icodextrin in the long-term treatment of refractory congestive heart failure. Perit Dial Int, 2009; 29: 116-118.

33. Wojtaszek E, Matuszkiewicz-Rowińska J, Grzejszczak A et al. Peritoneal ultrafiltration in the long-term treatment of heart failure refractory to pharmacological therapy. Perit Dial Int, 2010; 30: 39.

34. Sanchez JE, Ortega T, Rodriguez C et al. Efficacy of peritoneal utrafiltration in the treatment of refractory congestive heart failure. Nephrol Dial Transplant, 2010; 25: 605-610.

35. Ruhi C, Kocak H, Yavuz A, Süleymanlar G, Ersoy FF. Use of peritoneal ultrafiltration in the elderly refractory congestive heart failure patients. Int Urol Nephrol, 2012; 44: 963-969.

36. Diez Ojea B, Rodriguez Suárez C, Vidau P et al. Peritoneal dialysis role in heart failure treatment: experience in our center. Nefrologia, 2007; 27: 605-611.

37. Elhalel-Dranitzki M, Rubinger D, Moscovici A et al. CAPD to improve quality of life in patients with refractory heart failure. Nephrol Dial Transplant, 1998; 13: 3041-3042.

38. Sotirakopoulos NG, Kalogiannidou IM, Tersi ME, Mavromatidis KS. Peritoneal dialysis for patients suffering from severe heart failure. Clin Nephrol, 2011; 76: 124-129. 